\author{
Military Technical College \\ Kobry El-Kobbah, \\ Cairo, Egypt
}

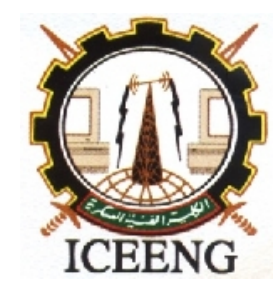

\title{
A modified PSO technique for electrical engineering applications
}

\author{
By \\ Magdi El-Saadawi \\ Ahmed Hassan \\ Mohammed Saeed ***
}

\section{$\underline{\text { Abstract: }}$}

PSO is a promising optimization technique proved a high performability as an evolutionary algorithm. Sometimes the conversion of that algorithm is not faster enough because the PSO initial setting values of that algorithm are chosen randomly and varies as long as the domain of application is varied. By means of classic PSO does not care about the limits of those random initial values which impacts the computation time. This paper presents a proposed approach that could be used to modify PSO technique which adapts the PSO initial values and number of particles for any optimization problem. The proposed modification makes the PSO technique faster and more applicable for electrical online applications. In most electrical applications, time factor is very important especially for on line applications. Protective devices coordination problem is a tedious and a time consuming task. To tackle that point of research, one of two cases has to be taken. First case is to redesign the coordination system according to any system changes. The second one is to maintain the old coordination system unchanged up to a specific DG penetration level. The new approach is used to solve the protective devices coordination problem in presence of DG in the two cases. A comparison between the classic PSO and the proposed modified one shows that the new approach gives fast results and shortens the computation time.

\section{Keywords:}

Distributed Generation, Particle Swarm Optimization (PSO), Coordination.

*** Electrical Engineering Department, Faculty of Engineering, Mansoura University, Mansoura, Egypt 


\section{Introduction:}

The basic PSO is a simple population based stochastic search evolutionary algorithm for global optimization. And many studies demonstrated that PSO converges fast and is robust, simple in implementation and use. While in spited of the merits, sometimes PSO may suffer from premature convergence and slowing down of convergence as the region of global optimum is approached [1]. To remedy the defects, a modified PSO is proposed. The proposed PSO modification cares about the determination of the PSO initial values limits and number of particles for any optimization problem. These values are chosen randomly in classic PSO technique which increases the computation time.

Research in power system has its share in applying PSO to various optimization problems. References [2-3] presented a review about the application of PSO technique in power system. Reference [4] proposed a Tabu Search with multi-objective PSO algorithm for optimal reactive power problem. This algorithm combines table search algorithm and mutation operator to select particle's global optimal solution. The authors in [5] used PSO to solve the optimal network reconfiguration problem for power loss reduction. They proposed decreasing the inertia weight linearly during the simulation. References [6-7] modified the PSO technique to solve the optimal power flow problem. The idea of this modification is based on this social behavior that each particle tries to leave its previous worst position and its group's previous worst position. The authors in [8] presented a global convergent PSO algorithm to solve the economic dispatch problem of power systems. They added some operators such as; mutation operator to ensure the algorithm search globally in theory; the neighborhood searching operator to improve the precision of convergence; and the disturbance operator makes it possible that the particle can jump out from local minima. For the optimal protective devices coordination problem, references [9-10] modified the PSO technique. The authors in [11] determined the number of particles and reported that five particles need 200 iterations for results convergence. The authors in [12] reported that instead of updating the entire particle's position in all D-dimensions at the same time, the positions are updated one after the other. Reference [13] proposed using the used the interior point method to obtain initial feasible solutions. This is done by initializing the pickup currents randomly, thus the problem becomes linear and the TDS values are calculated using the interior point method. The initial feasible solutions are then applied to the PSO algorithm.

This paper proposes a general approach to make the PSO computation time faster suitable for any online electrical application. Protective devices coordination problem is considered to be one of the most important electrical applications that need speed as the coordinated protective devices is set in an on-line manner. The new approach is used to solve the protective devices coordination problem in presence of DG by two cases. The first case is to redesign the coordination system according to any system changes. The 
second one is to maintain the old coordination system unchanged up to a specific DG penetration level. A comparison between the classic PSO and the proposed modified one shows that the new approach gives fast results and shortens the computation time.

\section{Classic Particle Swarm Optimization:}

PSO is initialized with a population of random solutions, called particles. Each particle represents a potential solution to the objective function under consideration. Each particle in the swarm can memorize its current position that is determined by evaluation of the objective function, velocity, and the best position visited during its flying tour in the problem search space referred to as "personal best position" (Pbest) [1]. For a minimization task, the position having a smaller function value is regarded to as having a higher fitness. Also the best position visited by all the particle is memorized, i.e., the best position among all Pbest positions referred to as "global best position" (gbest) [1]. At each time step (iteration) the velocity of each particle is updated using its current velocity and its position from Pbest and gbest according to the following equations:

$$
\begin{aligned}
& V_{i}(t+1)=\omega V_{i}(t)+r_{1} C_{1}\left(P_{i}(t)-X_{i}(t)\right)+r_{2} C_{2}\left(G_{i}(t)-X_{i}(t)\right) \\
& X_{i}(t+1)=X_{i}(t)+V_{i}(t+1)
\end{aligned}
$$

Where:

$$
\begin{array}{ll}
\mathrm{V}_{\mathrm{i}}(\mathrm{t}): & \text { represents the particles vector initial velocity } \\
\omega: & \text { inertia weight factor between two values }\left[\omega_{\min }: \omega_{\max }\right] \\
\mathrm{r}_{1} \& \mathrm{r}_{2}: & \text { random values in the range }[0: 1] \\
\mathrm{C}_{1} \& \mathrm{C}_{2}: & \text { positive constants } \\
\mathrm{P}_{\mathrm{i}}(\mathrm{t}): & \text { represents the best particles initial position } \\
\mathrm{X}_{\mathrm{i}}(\mathrm{t}): & \text { represents the particles vector initial position } \\
\mathrm{G}_{\mathrm{i}}(\mathrm{t}): & \text { represents the best initial position gives the best objective function } \\
& \text { among all particles } \\
\mathrm{V}_{\mathrm{i}}(\mathrm{t}+1): & \text { represents the particles vector updated velocity }
\end{array}
$$

\section{The Proposed Modified PSO Technique:}

As described in the previous section, PSO technique needs some vectors to be initialized randomly. The values of those vectors should be determined according to the nature of the problem to be solved. Authors propose a method to determine the optimal initial vectors to make PSO faster, performable, and accurate. Figure 1 shows the proposed modified PSO flowchart for any optimization problem for fast results convergence. First phase, any problem data must be collected in a vector and passed to the classic 
PSO program. These data include swarm parameters (number of particles, number of iteration ...etc), and the position vector of the problem .Second phase, the degree of convergence is tested on the obtained results. If the results converge, then the input system data is recorded and the PSO parameters are obtained. But if the result does not converge, the position vector limits is reduced and the number of particles is increased. Then, the new variables are passed again to the classic PSO program. This operation continues till the results converge.

Protective devices coordination problem is considered to be one of the most important electrical applications that need speed as the coordinated protective devices is set in an on-line manner. The new approach is used to solve the protective devices coordination problem in presence of DG by two cases. The first case is to redesign the coordination system according to any system changes. The second one is to maintain the old coordination system unchanged up to a specific DG penetration level.

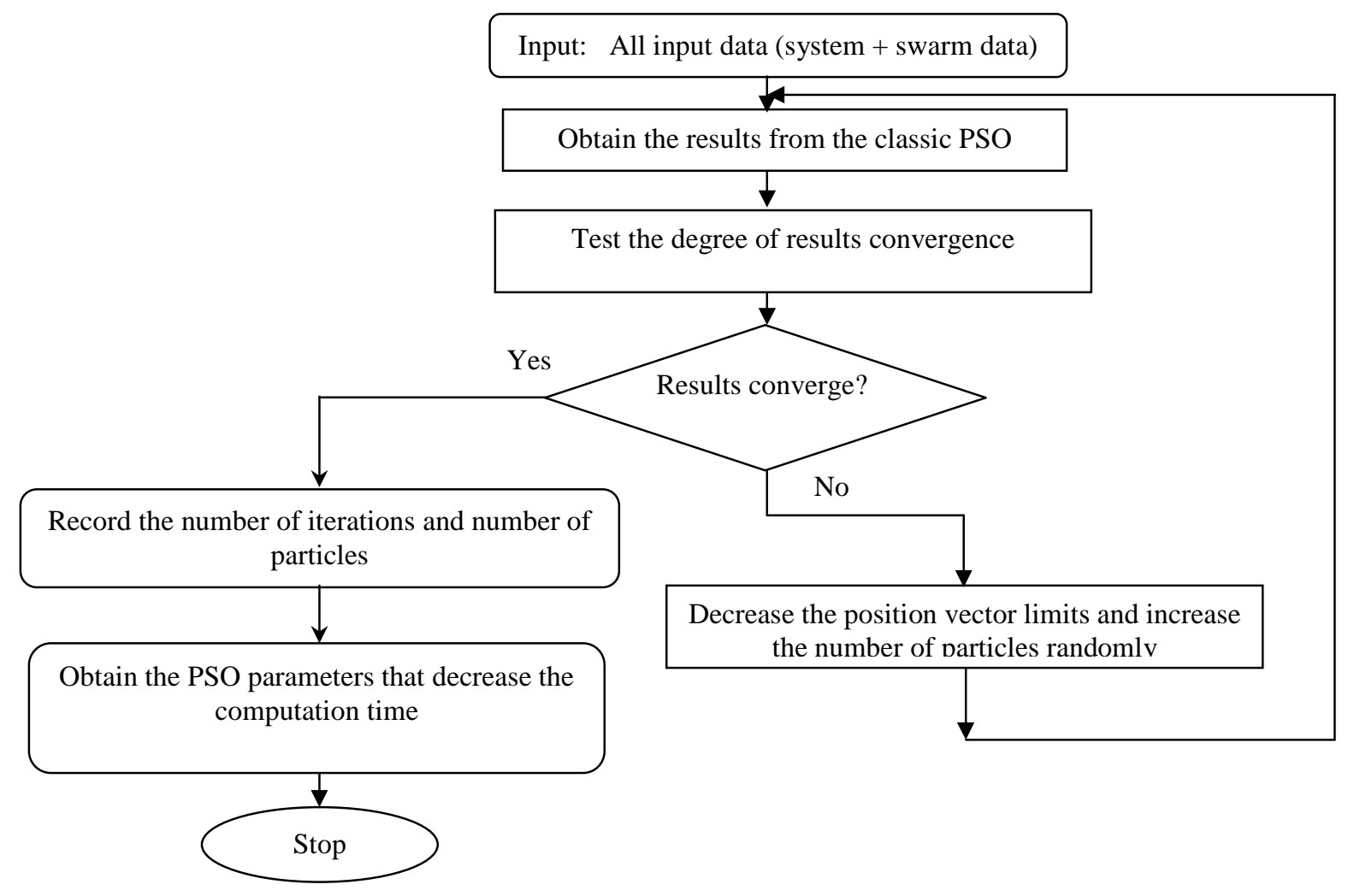

Figure (1): A general PSO modification flowchart for optimization problems 


\subsection{Case one: optimal initial values limits for coordination domain}

Time Dial Setting (TDS) adjusts the time delay before the relay operates whenever the fault current reaches a value equal to, or greater than, the relay current setting. The normal range for the TDS limits can be taken as (0.1pu:1pu) [13]. In the first technique, the proposed approach is used to determine the limits for the initial vector for TDS, and the number of particles. This modification provides better results in terms of the less computation time. The proposed modification is based on some computation trials in the normal TDS range $(0.1: 1)$ and changing the numbers of particles $(5,10,15, \ldots \ldots .$.$) as$ shown in Figure 2. In every computation trial, the number of iterations is determined. After 64 computation trial, the results converge fast with the limits of the initial vector for TDS is [0.1:0.38] with 100 particles. The maximum iteration number during the 64 computation trial is 73 iteration. The best selection for the initial vector limits reduces the maximum number of iterations to 20 . So, the computation time becomes more faster.

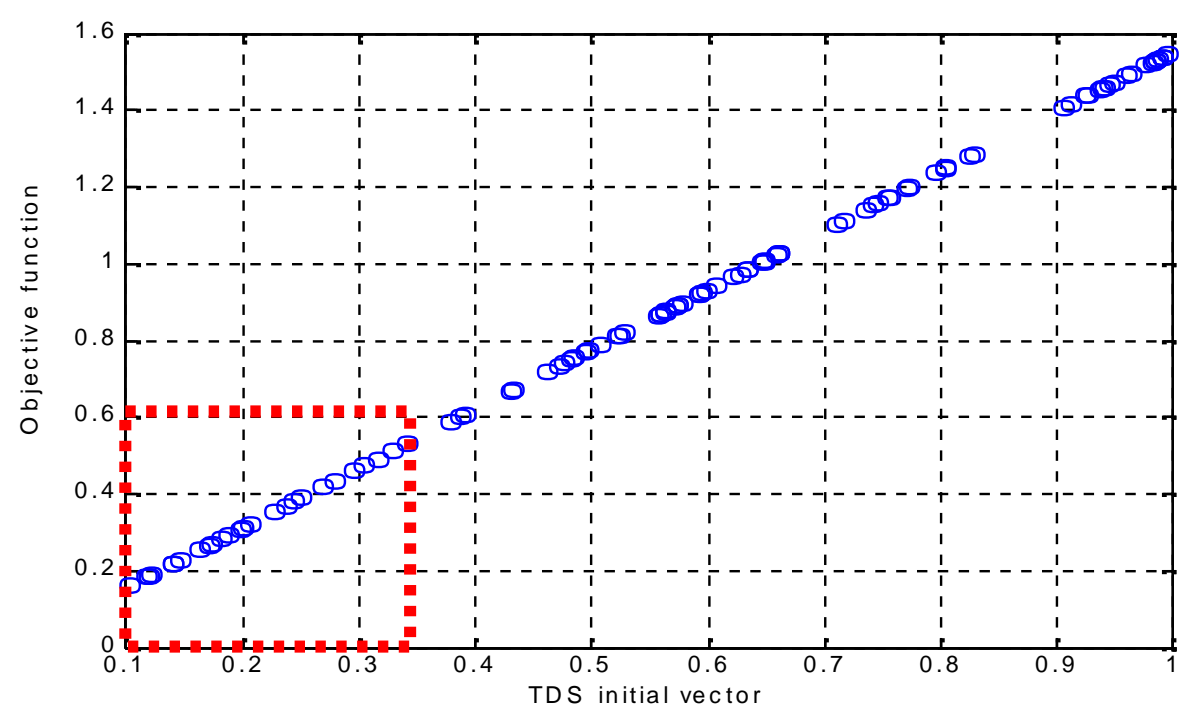

Figure (2): Determination of TDS initial vector limits

\subsection{Case two: optimal initial values limits for $D G$ penetration level}

In the second case, the proposed approach is used to determine the initial vector for DG current and the number of particles. This development provides better results in terms of the speed of convergence. The paper assumed that the normal range for the DG current limits can be taken as (0:0.5). The proposed development is based on some computation trials in the DG current range $(0: 0.5)$ and changing the numbers of particles $(5,10$, $15, \ldots \ldots \ldots$.$) . In every computation trial, the number of iterations is determined. After 41$ 
computation trial, the results convergence fast with the limits of the initial vector for DG current is [0.01:0.25] at 50 particle. The maximum iteration number during the 41 computation trial is 58 iteration. The best selection for the initial vector limits reduces the maximum number of iterations to 24 . So, the computation process more fast and accurate. Figure 3 shows the result for determination of DG current initial vector limits.

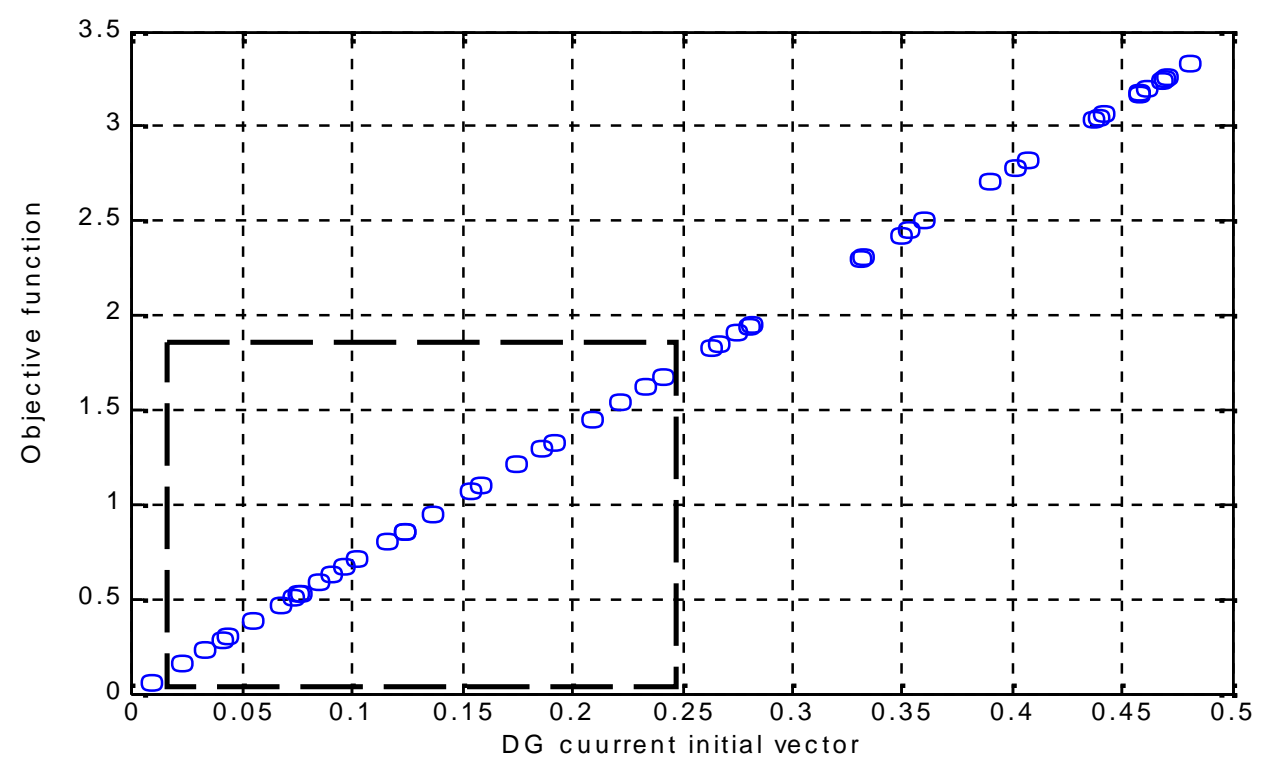

Figure (3): Determination of DG current initial vector limits

\section{Problem Formulation:}

As previously stated that the coordination problem in presence of DG is solved by two cases.

\subsection{Case one}

In this case, the coordination problem can be stated as a parametric optimization problem, where the objective function to be minimized is the sum of the operating times of the relays connected to the system. The problem can be formulated mathematically as in [15].

\subsection{Case two}

A comprehensive optimization function that can address the maximum size of the DG is formulated. The optimization problem is formulated considering some constraints such as; coordination criteria, system losses and voltage limit as follows: 


\section{Objective function:}

Max. $\left(\mathrm{P}_{\mathrm{DG}}\right)$

$P_{D G}=\sqrt{3} V_{i} I_{D G}$

Where: $\mathrm{P}_{\mathrm{DG}}$ : Output of DG, $\mathrm{V}_{\mathrm{i}}$ : The bus (i) voltage, $\mathrm{I}_{\mathrm{DG}}$ : The DG current

\section{Constraints:}

- Coordination constraint:

For traditional coordination scheme, the operating time for the main protective device is less than the operating time of the backup protective device. This time relation must be held in case of maintaining coordination after adding DG.

$$
T_{\text {main device }}-T_{\text {backup device }}<0
$$

- Voltage constraint:

$$
V_{i \min }<V_{i}<V_{i \max }
$$

- Losses constraint:

$$
\sum_{i=1}^{N} \text { Losses with } D G \leq \sum_{i=1}^{N} \text { Losses without } D G
$$

\section{Applications:}

\subsection{Case one}

\section{- RDTS-33 bus system:}

A developed Matlab program is implemented to the RDTS 33-bus test system [14] to calculate the optimal protective relays settings using classic PSO technique and proposed modified PSO technique under any system changes. These changes may be topological such as line outage or operational such as increasing the generating capacity. Figure 3 shows the RDTS 33 bus test system. 


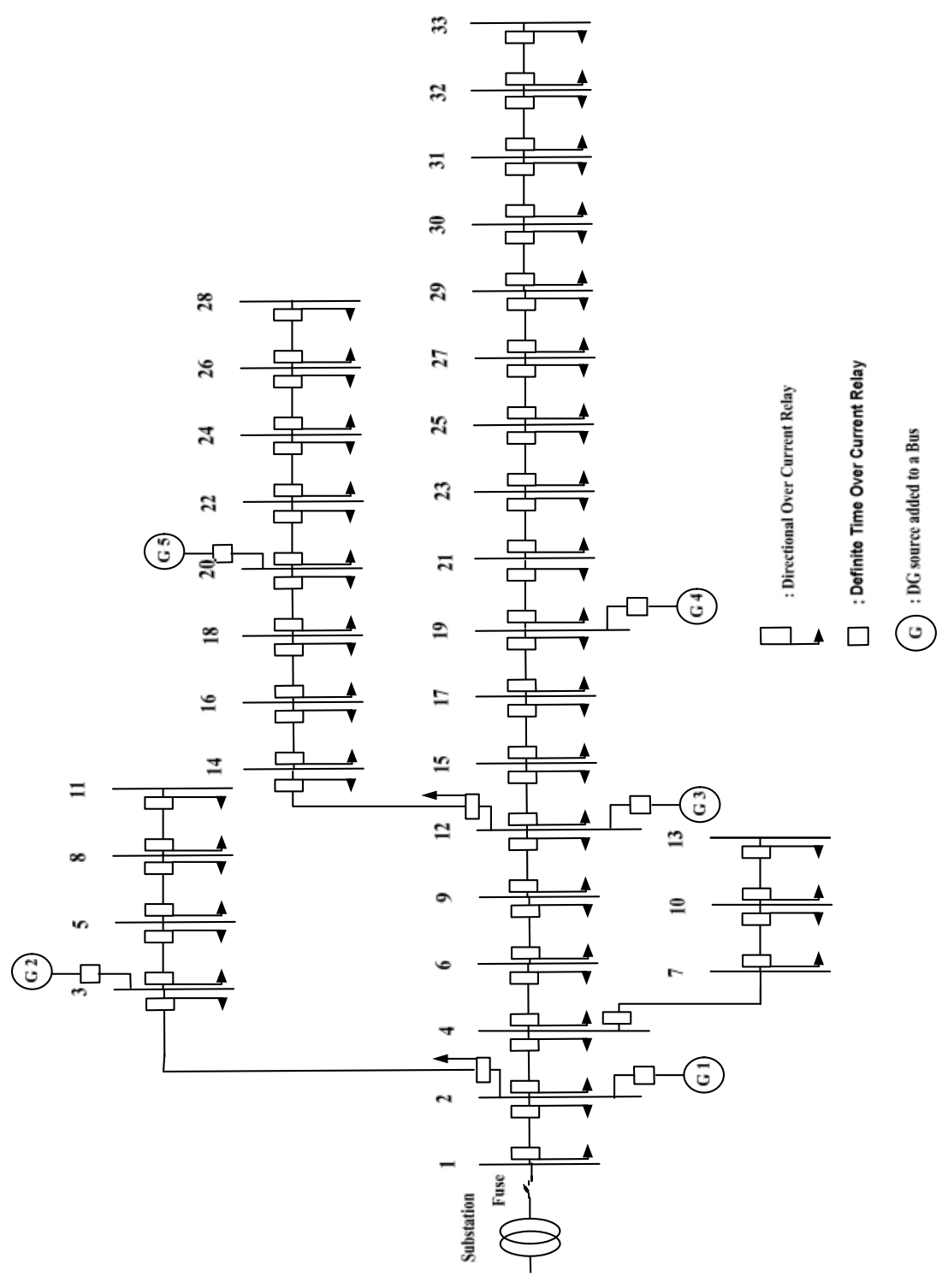

Figure (3): RDTS-33 bus test system

The program is implemented for different case studies as follows:

- Results with operational changes

The effect of the operational change on the relays settings can be shown by comparing the results of any relay of the system for different penetration level $(0,5,10,15,20,25$, and 30\%). Table 1 shows the design results of relay (6-9).

Figure 4 shows a comparison between the speed of computation in the classic PSO and the proposed modified technique. As shown from the Figure, the proposed modified PSO technique gives an accurate objective function in fast computation time. 
Table (1): Design results of relay (6-9) due to operational changes

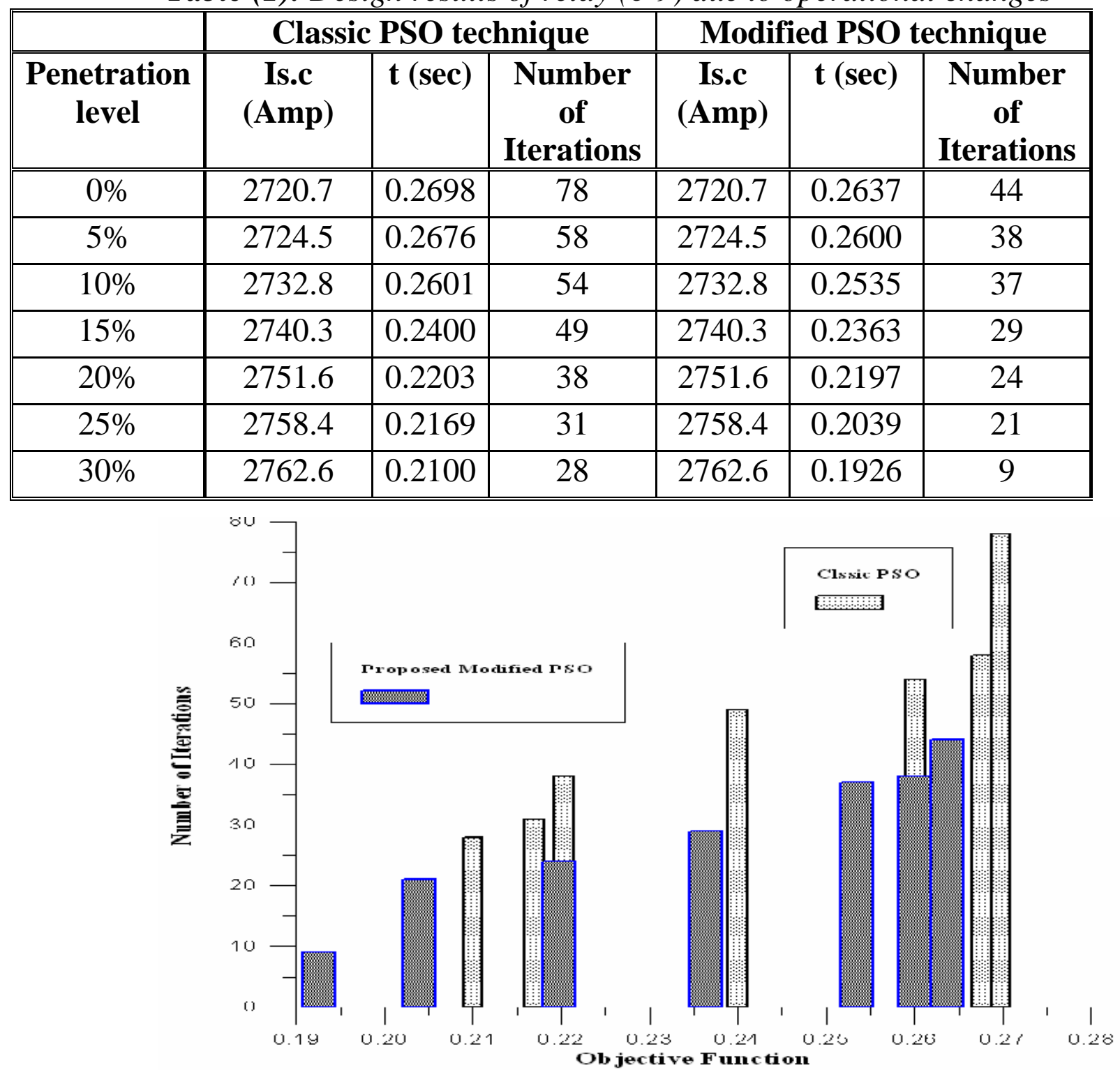

Figure (4): Comparison of computation speed in both techniques

\subsection{Case two}

- 10 bus system:

A test system [14] is shown in Figure 6 composed of a 3-phase 2.5 MVA, $11 \mathrm{KV}$ source supplying through a primary feeder, a 3-phase 2.5 MVA, 11000/400 V distribution transformer connected to two secondaries ended with 7 loads. The length of the primary feeder is $10 \mathrm{Km}$ and the length of the two secondaries is 6.5 $\mathrm{Km}$ with the following parameters: $\mathrm{R}=0.125 / \mathrm{Km}, \mathrm{L}=0.293 \mathrm{mH} / \mathrm{Km}$ and $\mathrm{C}=0.286 \mu \mathrm{F} / \mathrm{Km}$. 


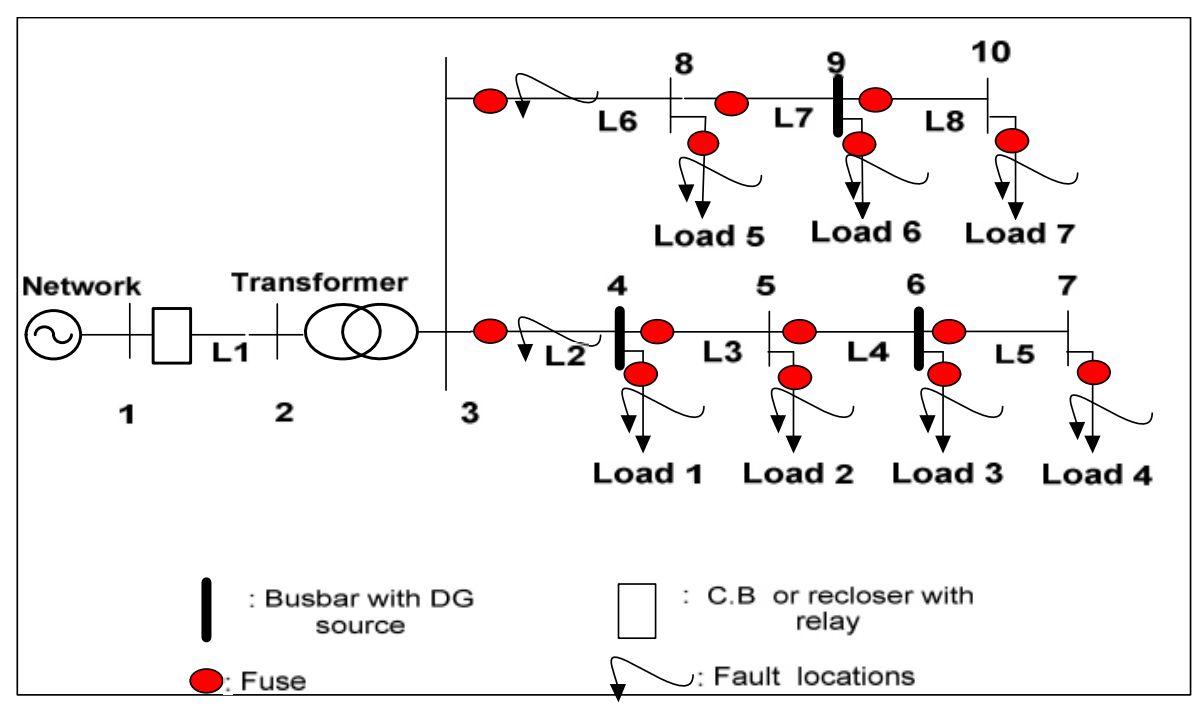

Figure (5): Schematic diagram for the 10 bus system

\section{- Results}

The classic PSO is applied to this system before applying the modified PSO technique. Table 2 shows the results comparison between the classic PSO technique and the proposed modified PSO technique. From the table; the maximum DG penetration level that maintains the traditional protective devices coordination unchanged occurs at bus 3 . The classic PSO calculates this value as 0.4305 MW (17.22\% penetration level) in 47 iteration. But the proposed modified PSO gives this value as $0.4123 \mathrm{MW}(16.491 \%$ penetration level) in 18 iteration. The obtained value using the modified PSO technique is more accurate in less computation time.

Table (2): Results comparison between classic PSO and modified PSO for 10-bus feeder

\begin{tabular}{|c|c|c|c|c|c|c|}
\hline & \multicolumn{3}{|c|}{ Classic PSO technique } & \multicolumn{3}{c|}{ Modified PSO technique } \\
\hline $\begin{array}{c}\text { Bus } \\
\text { Number }\end{array}$ & $\begin{array}{c}\text { Minimum } \\
\text { DG Size } \\
\text { (MW) }\end{array}$ & $\begin{array}{c}\text { Penetration } \\
\text { level } \\
(\mathbf{\%})\end{array}$ & $\begin{array}{c}\text { Number } \\
\text { of } \\
\text { Iterations }\end{array}$ & $\begin{array}{c}\text { Minimum } \\
\text { DG Size } \\
\text { (MW) }\end{array}$ & $\begin{array}{c}\text { Penetration } \\
\text { level } \\
\text { (\%) }\end{array}$ & $\begin{array}{c}\text { Number } \\
\text { of } \\
\text { Iterations }\end{array}$ \\
\hline 3 & 0.4305 & 17.22 & 47 & 0.4123 & 16.491 & 18 \\
\hline 4 & 0.4216 & 16.864 & 21 & 0.3925 & 15.700 & 11 \\
\hline 5 & 0.4290 & 17.16 & 41 & 0.3947 & 15.788 & 16 \\
\hline 6 & 0.4138 & 16.552 & 33 & 0.4000 & 16.000 & 21 \\
\hline 7 & 0.4220 & 16.88 & 31 & 0.4106 & 16.424 & 20 \\
\hline 8 & 0.4153 & 16.612 & 28 & 0.4118 & 16.472 & 17 \\
\hline
\end{tabular}




\section{Conclusion:}

PSO technique is a novel intelligent computation optimization method. It is an effective evolutionary technique and easy to implement. PSO initial setting values are chosen randomly and changes from domain of application to another which increases the computation time. This paper presented a new approach to modify PSO based on a prediction technique to determine the PSO initial values and number of particles for any optimization problem. The proposed modification makes the PSO technique faster and more applicable for electrical online applications. The new approach is used to solve the protective devices coordination problem in presence of DG. A comparison between the classic PSO and the proposed modified one shows that the new approach gives fast results and shortens the computation time.

\section{References:}

[1] M. Clerc, "Particle Swarm Optimization", Book, Antony Rowe Ltd, United Kingdom, 2006.

[2] M. Alrashidi, M. EL-Hawary, "A Survey of Particle Swarm Optimization Applications in Power System Operations, March 2006.

[3] D. Saxena, S. Singh and K. Verma , "Application of computational intelligence in emerging power systems", International Journal of Engineering, Science and Technology, Vol. 2, No. 3, P. 1-7, 2010.

[4] J.Wu Nan, et al., "Research on Multi-Objective reactive power optimization based on modified Particle Swarm Optimization algorithm", Control and Decision Conference, P. 477-480, 26-28 May 2010.

[5] A. Y. Abdelaziz, et al., "A Modified Particle Swarm Technique for Distribution Systems Reconfiguration", The Online Journal on Electronics and Electrical Engineering (OJEEE) Vol. 1, No.2, P. 121129.

[6] H.Hoseinabadi, H. Hosseini, and S.H. Hajian, "Optimal power flow solution by a modified particle swarm optimization algorithm", The 43rd International Universities Power Engineering Conference, UPEC, P. 1-4,Sept. 2008.

[7] R.Cui, et al., "A modified particle swarm optimization algorithm and its application in optimal power flow problem", International Conference on Machine Learning and Cybernetics, Vol.5, P. 28852889,18-21 Aug. 2005.

[8] Y.He Hou, et al., "Economic Dispatch of Power Systems Based on 
the Modified Particle Swarm Optimization Algorithm", Transmission and Distribution Conference and Exhibition: Asia and Pacific, P. 1-6, 2005.

[9] H.Zeineldin, E. EI-Saadany, and M. Salama., "Optimal coordination of overcurrent relays using a modified particle swarm optimization", Electric Power Systems Research, Vol.76, P. 988-995, 2006.

[10] D. Vijayakumar and R. K. Nema , "A Novel Optimal Setting for Directional over Current Relay Coordination using Particle Swarm Optimization", International Journal of Electrical Power and Energy Systems Engineering, 2008.

[11] M. Mansour, F. Mekhamer, and N.El-Sherif , "A Modified Particle Swarm Optimizer for the Coordination of Directional Overcurrent Relays", IEEE Transaction on Power Delivery, Vol. 22, No. 3, July 2007.

[12] N. Nimpitiwan, et al., "Consequences of Fault Currents Contributed by Distributed Generation", Report, Power Systems Engineering Research Center, June 2006.

[13] A.J. Urdaneta, R. Nadira, and L.G Jimerez: "Optimal Coordination of Directional Overcurrent Relays in Interconnected Power Systems" IEEE Transaction on Power Delivery, Vol. 3, No. 3, P. 903 - 911, July 1988.

[14] M. S. Kandil, M. G. Osman, M. M. El-Saadawi and M. A. Saeed, "Determination of Maximum DG Penetration Level that Maintain Protective Devices Coordination Unchanged: A Framework", The 3rd International Power Engineering and Optimization Conference, PEOCO2009, Selangor, Malaysia, 3-4 June 2009.

[15] M. El-Saadawi, A. Hassan, M. Saeed, "Adaptive Optimal Relay Coordination Scheme for Distributed Generation", International Journal of Distributed Energy Resources, Vol. 7, No. 2, P. 79-91. March. 2011. 